\title{
THE POSSIBLE BELTS FOR EXTRASOLAR PLANETARY SYSTEMS
}

\author{
Ing-Guey Jiang, ${ }^{1}$ M. Duncan, ${ }^{2}$ and D. N. C. $\operatorname{Lin}^{3}$ \\ RESUMEN
}

Desde la década de los 90 se han descubierto más de 100 planetas extrasolares. A diferencia del Sistema Solar, estos planetas tienen excentricidades en un amplio intervalo, desde 0 hasta 0.7. El primer objeto del Cinturón de Kuiper se descubrió en 1992. Se plantea la cuestión de si los sistemas planetarios extrasolares podrían tener estructuras como el Cinturón de Kuiper o el de los asteroides. Investigamos la estabilidad de estos sistemas para distintas excentricidades con los métodos de Rabl \& Dvorak (1988) y Holman \& Wiegert (1999). Sostenemos que la mayor parte de los sistemas planetarios extrasolares pueden tener cinturones en las regiones externas. No obstante, encontramos que las órbitas de gran excentricidad son muy efectivas para destruir estas estructuras.

\section{ABSTRACT}

More than 100 extrasolar planets have been discovered since the 1990s. Unlike those of the solar system, these planets' orbital eccentricities cover a huge range from 0 to 0.7 . Incidentally, the first Kuiper belt object was discovered in 1992. Thus an interesting and important question will be whether extrasolar planetary systems could have structures like the Kuiper belt or asteroid belt. We investigate the stability of these planetary systems with different orbital eccentricities by similar procedures to Rabl \& Dvorak (1988) and Holman \& Wiegert (1999). We claim that most extrasolar planetary systems can have their own belts at the outer regions. However, we find that orbits with high eccentricity are very powerful in depletion of these populations.

Key Words: STARS: PLANETARY SYSTEMS

\section{INTRODUCTION}

In recent years, the number of discovered extrasolar planets is increasing quickly due to astronomers' observational effort and therefore interest in dynamical study in this field has been renewed.

These discovered planets, with masses from 0.16 to 17 Jupiter masses $\left(M_{J}\right)$, have semimajor axes from $0.04 \mathrm{AU}$ to $4.5 \mathrm{AU}$ and also a wide range of eccentricities. Moreover, there is a mass-period correlation for discovered extra-solar planets, which gives a paucity of massive close-in planets. Jiang, Ip \& Yeh (2003) claimed that although tidal interaction could explain this paucity (Pätzold \& Rauer 2002), the mass-period correlation might be weaker at the time when these planets were just formed. Gu, Lin \& Bodenheimer (2003) and Sasselov (2003) also have done very interesting work on close-in planets. Therefore, some of these extrasolar planets' dynamical properties are very different from the planets in the solar system.

Nevertheless, similarities between extrasolar and solar planets do exist. For example, there is a new discovery about a Jupiter-like orbit very recently, i.e. a Jupiter-mass planet on a circular long-period orbit

\footnotetext{
${ }^{1}$ National Central Univ., Taiwan.

${ }^{2}$ Queen's University, Canada.

${ }^{3}$ University of California, USA.
}

(semimajor axis $a=3.65 \mathrm{AU}$ ) was detected.

On the other hand, some planetary systems were claimed to have discs of dust and they are regarded as young analogues of the Kuiper belt. For example, Greaves et al. (1998) found a dust ring around a nearby star $\epsilon$ Eri and Jayawardhana et al. (2000) detected the dust in the 55 Cancri planetary system. Particularly, the $\beta$ Pictoris planetary system has a warped disc and the influence of a planet might explain this warp (Augereau et al. 2001).

Given the fact that many extrasolar planets' orbital eccentricities are very big and some of them still could have analogues of the Kuiper belt, it would be interesting to investigate in what environments and conditions the belts could exist for a planetary system. Following Rabl \& Dvorak (1988) and Holman $\&$ Wiegert (1999), we use the critical semimajor axis as a tool to explore the unstable zone where it would be difficult for a belt to exist for a given planetary system.

We will explain the basic model in Section 2. In Section 3, we study the cases of one planet. We discuss multiple planetary systems in Section 4 and the effect of a companion star in a binary system in Section 5. We make conclusions and also discuss the possible implications in Section 6. 


\section{THE MODEL}

A direct force integration of the equation of motion is required for the computation of the orbital evolution of our systems. We adopt a numerical scheme with Hermite block-step integration which has been developed by Sverre Aarseth (Markino \& Aarseth 1992, Aarseth, Lin \& Palmer 1993).

We consider a range of ratio $\left(\mu=M_{p} /\left(M_{p}+M_{*}\right)\right)$ of masses $\left(M_{p}\right.$ and $\left.M_{*}\right)$, where $M_{p}$ is the planetary mass and $M_{*}$ is the mass of the central star. We also consider a range of orbital eccentricity $\left(e_{p}\right)$. The semimajor axis of the (inner) planet is set to be unity for systems with one (two) planet(s) such that all other lengths are scaled with its physical value. We adopt $G\left(M_{*}+M_{p}\right)=1$ so that the planetary orbital period is $2 \pi$.

We mainly determine the inner and outer critical semimajor axis, i.e. the innermost and outermost semimajor axes at which the test particles at both $\theta=0^{\circ}, 90^{\circ}$ survive. The definition of survival here is that the distance between the test particle and the central star must be smaller than a critical value $R_{d}$ during a time $T_{d}$. The value of $R_{d}$ is arbitrarily set to be 3 times the planetary initial semimajor axis and we choose $T_{d}=2 \pi \times 10^{4}$. Therefore, more precisely, the inner critical semimajor axis is: within the region between the planet and central star, the outermost semimajor axis that a test particle can survive for $T_{d}$, and the outer critical semimajor axis is: out of the region between the planet and central star, the innermost semimajor axis that a test particle can survive for $T_{d}$.

Based on several test runs, we find that the value of $a_{c}$ does not change significantly if $T_{d}$ is increased to $2 \pi \times 10^{6}$. That is, planets which can survive for $2 \pi \times 10^{4}$ can usually remain attached for a much longer timescale. Thus, we find the critical semimajor axis $a_{c}$ to be a useful parameter to classify our results (Dvorak et al. 2004).

\section{THE SYSTEMS OF ONE PLANET}

We determine both the inner and outer critical semimajor axes for a system with one planet moving around the central star. The area between inner and outer critical semimajor axes can be regarded as an "unstable zone". We get the width of the unstable zone by subtracting the value of the inner critical semimajor axis from the value of the outer one.

We determine these critical semimajor axes for different planetary masses. We also consider different eccentricities of the planet's orbits, which vary from $e=0$ to $e=0.8$. The results are in Tables 1a-c.
TABLE $1 \mathrm{~A}$

Critical Semimajor Axis When $\mu=0.005$

\begin{tabular}{lcc}
\hline & inner & outer \\
\hline$e=0.0$ & 0.7 & 1.5 \\
$e=0.2$ & 0.5 & 1.9 \\
$e=0.4$ & 0.3 & 2.1 \\
$e=0.6$ & 0.2 & 2.2 \\
$e=0.8$ & 0.1 & 2.5 \\
\hline
\end{tabular}

TABLE 1B

Critical Semimajor Axis When $\mu=0.001$

\begin{tabular}{lcc}
\hline & inner & outer \\
\hline$e=0.0$ & 0.8 & 1.3 \\
$e=0.2$ & 0.6 & 1.6 \\
$e=0.4$ & 0.4 & 1.8 \\
$e=0.6$ & 0.2 & 2.1 \\
$e=0.8$ & 0.1 & 2.2 \\
\hline
\end{tabular}

TABLE 1C

Critical Semimajor Axis When $\mu=0.0001$

\begin{tabular}{lcc}
\hline & inner & outer \\
\hline$e=0.0$ & none & none \\
$e=0.2$ & 0.7 & 1.3 \\
$e=0.4$ & 0.5 & 1.6 \\
$e=0.6$ & 0.3 & 1.8 \\
$e=0.8$ & 0.1 & 1.9 \\
\hline
\end{tabular}

If the mass of the central star is assumed to be $1 M_{\odot}$, the planet has about $5 M_{J}$ for the results in Table 1a and has about $1 M_{J}$ for the results in Table 1b. From Tables $1 \mathrm{a}$ and $1 \mathrm{~b}$, we find that the results are quite similar for these two cases. Approximately, the inner critical semimajor axis is about $3 / 4$ and the outer critical semimajor axis is about $3 / 2$ when the eccentricity $e=0$. After we increase the eccentricity, the inner critical semimajor axis becomes about $(1-e) 3 / 4$ and the outer critical semimajor axis becomes about $(1+e) 3 / 2$. This is reasonable because the pericentre is at $(1-e) a$ and the apocentre is at $(1+e) a$ where $a$ is the semimajor axis of the planet and thus the planet's orbit covers a larger radial range, the critical semimajor axis should change correspondingly.

However, from the results in Table 1c, when the mass of the planet is much less (one order less) than $M_{J}$, the planet depletes nothing and thus both the inner and outer critical semimajor axes do not exist in the case of zero eccentricity. Interestingly, when we increase the eccentricity, the effect of eccentricity gradually dominates and critical semimajor axes can become of similar order to the ones in Tables 1a and $1 \mathrm{~b}$ even if the mass of the planet is much less. 


\section{THE SYSTEMS OF TWO PLANETS}

Interestingly, there are two belts of small bodies in the solar system and these two are located in very different environments: the asteroid belt is between two planets and the Kuiper belt is located at the outer part of the planetary disc. Therefore, it will be important to study multiple planetary systems and determine the physical locations where we can possibly have stable belts.

To simplify the model and as a first step, we choose the case of two planets, both with mass about $M_{J}$, i.e. $\mu=0.001$. The inner planet will be planet 1 and the outer planet will be planet 2 hereafter. The stability of this system depends on their separation and also orbital eccentricities. To reduce the parameters, we always set the initial eccentricity of planet 2 to be zero but study the effect of different initial eccentricity of planet 1 only.

These two planets are in fact interacting with each other. When the initial eccentricity of planet 1 is small, the interaction is weaker and planet 2 stays moving on a nearly circular orbit. When the initial eccentricity of planet 1 is bigger, the interaction becomes much stronger and the eccentricity of planet 2 gradually increases in our simulations.

We checked the critical semimajor axis of planet 2 for different eccentricities of planet 1 and we found that when planet 1 is initially located at $r=1$, planet 2 should be at about $r=3$ to make the system stable during $10^{4}$ orbital periods of planet 1.

Therefore, we set the semimajor axis of planet 1 to be unity, the semimajor axis of planet 2 to be 3 and both at $\theta=0^{\circ}$ initially. We then begin to place test particles to determine the inner and outer critical semimajor axes for both planets. Tables 2a-b are the results.

When the eccentricity is 0 or 0.2 , we found that there could be an asteroid belt-like population between these two planets. The system is quite stable and thus the results in last section, i.e. Table $1 \mathrm{~b}$, gives us good hints for the size of the unstable zone around planet 1 though the unstable zone does expand a bit for this system with two planets. However, when the eccentricity is larger, there is no stable zone between these two planets and the most possible location to have a belt is outside outer critical semimajor axis of planet 2. This result tells us that if the eccentricities of the planets in the solar system were not between 0 and 0.2 , but much larger, it is unlikely that there would be an asteroid belt.

\section{THE EFFECT OF A COMPANION STAR}

Some of the host stars of the discovered planetary systems are indeed members of binary systems, for
TABLE 2A

\begin{tabular}{|c|c|c|}
\hline \multicolumn{3}{|c|}{ Critical Semimajor Axis of Planet 1} \\
\hline & inner & outer \\
\hline$e=0.0$ & 0.7 & 1.3 \\
\hline$e=0.2$ & 0.6 & 1.7 \\
\hline$e=0.4$ & 0.3 & none \\
\hline$e=0.6$ & 0.2 & none \\
\hline$e=0.8$ & 0.1 & none \\
\hline \multicolumn{3}{|c|}{ TABLE 2B } \\
\hline & inner & outer \\
\hline$e=0.0$ & 2.3 & 3.9 \\
\hline$e=0.2$ & 2.3 & 3.9 \\
\hline$e=0.4$ & none & 3.9 \\
\hline$e=0.6$ & none & 3.9 \\
\hline$e=0.8$ & none & 7.5 \\
\hline
\end{tabular}

example, 16 Cyg B, $55 \rho^{1}$ Cnc, $\tau$ Boo.

It will be interesting to see the effect of a secondary companion star on the planetary system in which a planet moves around the binary primary. Thus, assuming an equal mass binary, we determine the critical semimajor axis of the binary secondary for the cases that the eccentricity of the binary $e_{\mathrm{b}}$ is 0.2 and 0.6. We assume the planet has mass about $1 M_{J}$, the initial eccentricity ranges from 0 to 0.8 with respect to the binary primary. Both the binary secondary and the planet begin from $\theta=0^{\circ}$. Tables $3 \mathrm{a}-\mathrm{b}$ are our results. Most of the discovered planets are stable since their binary separations are much bigger than the critical semimajor axes.

On the other hand, the binary might affect the extension of possible belt populations. To investigate this point further, we now use two test particles (at $\theta=0^{\circ}$ and $90^{\circ}$ ) to determine the critical semimajor axis of the binary. We find that the critical semimajor axis of the system becomes bigger in order to make the test particles survive. For the case of $e_{\mathrm{b}}=0.2$, the critical semimajor axis $a_{\mathrm{b}}$ is about 20. For $e_{\mathrm{b}}=0.6, a_{\mathrm{b}}$ is about 36 . This result does not depend on the details of other parameters such as the initial eccentricity of the planet or the semimajor axis of the test particles. Because the critical semimajor axis becomes much bigger, the presence of a binary secondary might affect the extension or even existence of possible Kuiper belt populations.

\section{CONCLUSIONS AND IMPLICATIONS}

We have studied the possible conditions for which a belt could be stable and thus exist for assumed planetary systems. Because we explore different eccentricities for the given planetary systems, our re- 
Table 3a

\begin{tabular}{lc}
\multicolumn{2}{c}{ Critical Semimajor Axis When $e_{\mathrm{b}}=0.2$} \\
\hline & $a_{\mathrm{b}}$ \\
\hline$e=0.0$ & 4.1 \\
$e=0.2$ & 4.5 \\
$e=0.4$ & 4.9 \\
$e=0.6$ & 5.2 \\
$e=0.8$ & 5.4 \\
\hline
\end{tabular}

Table $3 b$

Critical Semimajor Axis When $e_{\mathrm{b}}=0.6$

\begin{tabular}{lc}
\hline & $a_{\mathrm{b}}$ \\
\hline$e=0.0$ & 9.7 \\
$e=0.2$ & 10.1 \\
$e=0.4$ & 10.6 \\
$e=0.6$ & 11.1 \\
$e=0.8$ & 11.5 \\
\hline
\end{tabular}

sults should be applicable to discovered extrasolar planetary systems. In addition to those systems with one planet, systems with two planets are studied and the effect of a companion star is also investigated.

We find that highly eccentric orbits are very powerful in depletion of belt-like populations such as the asteroid belt and the companion star might restrict the extension of such populations.

On the other hand, as emphasized by Yeh \& Jiang (2001), the planet should dynamically couple with the belt over the evolutionary history. That is, the planet's mass and orbital properties would determine the existence and affect the position of the belt, but if the belt is massive enough, it will in turn influence the planet, too. This is particularly important during the early stage of planetary formation since the circumstellar belt is more massive then. For example, Jiang \& Ip (2001) noted that interaction with the belt could bring the planetary system of $v$ And to its current orbital configuration.

Moreover, according to Jiang \& Yeh (2004), the probability that the planet moves stably around the outer edge is much smaller than near the inner edge. This conclusion is consistent with the principal result in Jiang \& Yeh (2003).

What could we learn for the solar system from their theoretical result? From the observational picture of the asteroid belt, we know that: (a) the outer edge looks more diffuse and (b) Mars is moving stably close to the inner edge of the asteroid belt but Jupiter is quite far from the outer edge. One possible explanation is that Jupiter is much more massive and thus those planetesimals close to Jupiter would have been scattered away during the formation of the solar system. If we apply the model of Jiang \& Yeh (2004) to the asteroid belt and the point mass which represents the planet in their model can also represent larger asteroids, their theoretical result provides another choice to explain both (a) and (b).

It is known that there is another belt in the solar system, the Kuiper belt, after the first object was detected (Jewitt \& Luu 1993). Allen, Bernstein \& Malhotra (2001) did a survey and found that they could not find any Kuiper belt objects (KBOs) larger than $160 \mathrm{~km}$ in diameter beyond $50 \mathrm{AU}$ in the outer solar system. If we apply the model of Jiang \& Yeh (2004) to this problem and the point mass which represents the planet in their model now represents larger KBOs moving within the Kuiper belt, we find that their theoretical results provide a natural mechanism to do this orbit rearrangement: larger KBOs might have been moving towards the inner edge of the belt due to the influence from the belt.

\section{REFERENCES}

Aarseth, S. J., Lin, D. N. C., \& Palmer, P. L., 1993, ApJ, 403,351

Allen, R. L., Bernstein, G. M., \& Malhotra, R., 2001, ApJ, 549, 241

Augereau, J. C., Nelson, R. P., Lagrange, A. M., Papaloizou, J. C. B., \& Mouillet, D., 2001, A\&A, 370, 447

Dvorak, R., Pilat-Lohinger, E., Bois, E., Funk, B., Freistetter, F., \& Kiseleva-Eggleton, L. 2004, this volume

Greaves, J. S. et al., 1998, ApJ, 506, L133

Gu, P.-G., Lin, D. N. C., \& Bodenheimer, P. H., 2003, ApJ, 588, 509

Holman, M. J., \& Wiegert, P. A., 1999, AJ, 117, 621

Jayawardhana, R., Holland, W. S., Kalas, P., Greaves, J. S., Dent, W. R. F., Wyatt, M. C., \& Marcy, G. W. 2002, ApJ, 570, L93

Jewitt, D., \& Luu, J. X., 1993, Nature, 362, 730

Jiang, I.-G., \& Ip, W.-H., 2001, A\&A, 367, 943

Jiang, I.-G., Ip, W.-H., \& Yeh, L.-C., 2003, ApJ, 582, 449

Jiang, I.-G., \& Yeh, L.-C., 2003, Int. J. Bifurcation and Chaos, 13, 534

Jiang, I.-G., \& Yeh, L.-C., 2004, Int. J. Bifurcation and Chaos, in press, astro-ph/0309220

Makino, J., \& Aarseth, S., J., 1992, PASJ, 44, 141

Pätzold, M., \& Rauer, H., 2002, ApJ, 568, L117

Rabl, G., \& Dvorak, R., 1988, A\&A, 191, 385

Sasselov, D. D., 2003, ApJ, 596, 1327

Yeh, L.-C., \& Jiang, I.-G., 2001, ApJ, 561, 364

Ing-Guey Jiang: Institute of Astronomy, National Central University, Taiwan (jiang@astro.ncu.edu.tw).

M. Duncan: Department of Physics, Queen's University, Kingston, ON K7L 3N6, Canada

(duncan@astro.queeensu.ca).

D.N.C. Lin: UCO/Lick Observatory, University of California, Santa Cruz, CA 95064, USA (lin@ucolick.org). 


\section{DISCUSSION}

Scarfe - What units are your belt masses expressed in?

Jiang - In units of a solar mass.

Sterzik - In order to confirm putative belts around extra-solar planet systems, what wavelength regimes would you recommend? In other words, what radii and temperatures do you expect to have stable configurations?

Jiang - Because the current results have been detected in the far infrared, this is probably the right choice.

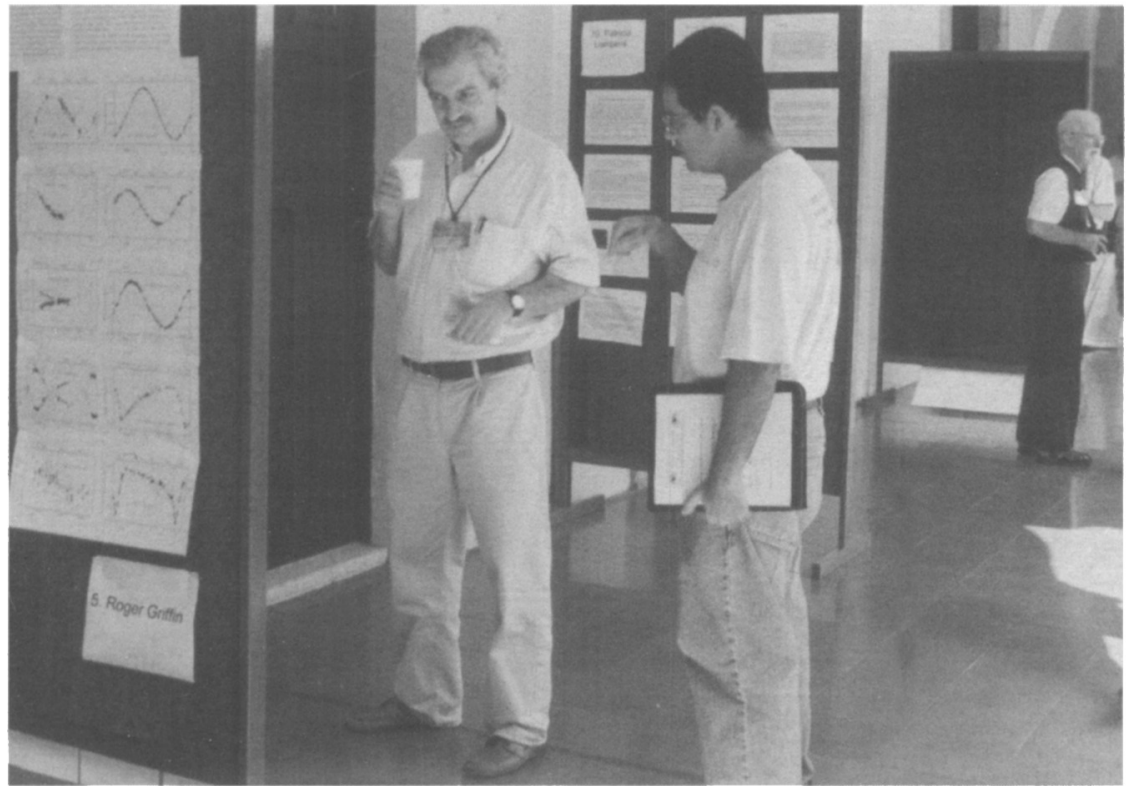

Guillem Anglada and Abraham Luna.

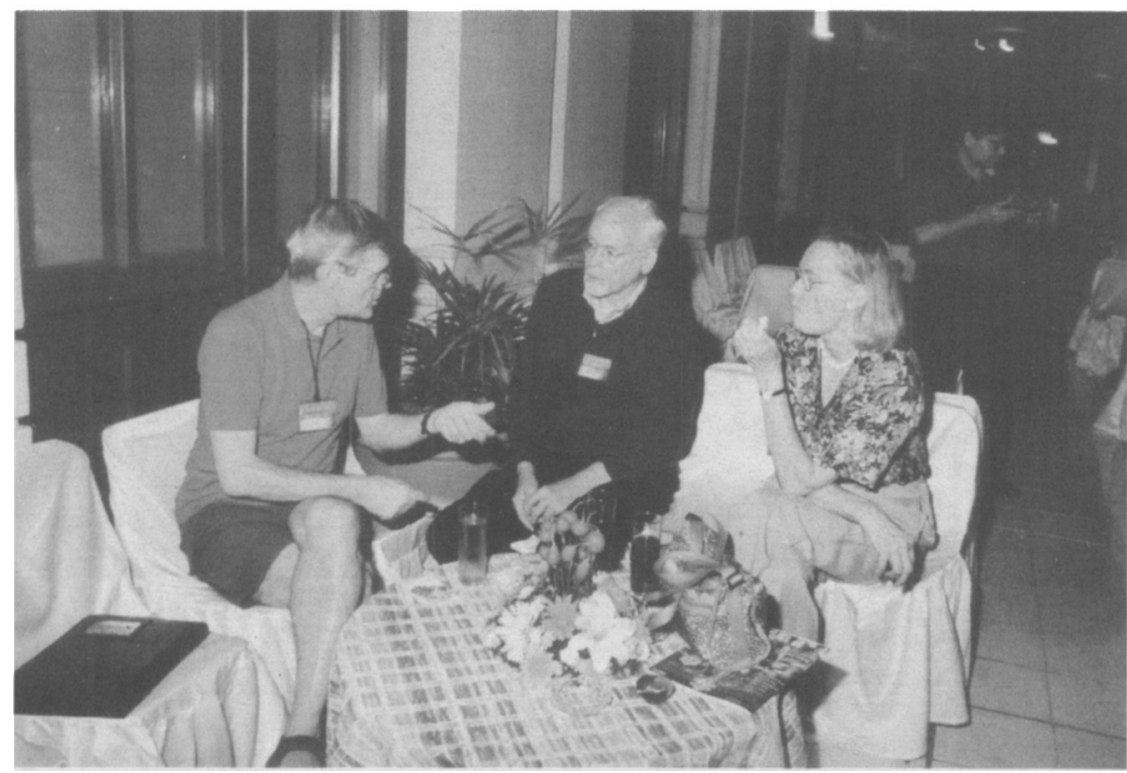

Hugo Levato and A. G. Davis Philip. 